\title{
Vehicular Adaptive Cruise Control Using Laguerre Functions Model Predictive Control
}

\author{
Dileep K ${ }^{\# 1}$, Sreedeep Krishnan ${ }^{\# 2}$, Akhil Jose ${ }^{\# 3}$ \\ \#1-3 Assistant Professor, AE\&I Dept, ASIET, Kalady, Kerala, India \\ ${ }^{1}$ dileep03k@gmail.com, ${ }^{2}$ sreedeepkrishnan@gmail.com \\ 3 akhilvjose@gmail.com
}

\begin{abstract}
Adaptive cruise control (ACC) is an improved form of cruise vehicle where the vehicle will automatically follow the preceding vehicle at a desired speed keep a safe distance between the vehicles in all traffic scenarios. Additional to this the ACC vehicle guarantees safe vehicle tracking, good fuel efficiency by avoiding unwanted acceleration and breaking and high driver comfort by reducing the jerk to minimum. The designed controllers controls and optimizes the acceleration, engine power and breaking so that the required objectives are achieved. A two level hierarchical control architecture is used in which the nonlinear dynamics of the vehicle is compensated in the lower level controllerwere throttle power is controlled using a PID controller and brake pressure is controlled using a feedback controller. Upper level controller gives the required input commands to the lower level controller. Upper level controller is designed using a model predictive control (MPC) which generated a control signal determining the longitudinal acceleration based on the inter-vehicular distance and velocity. MPC generates a cost function considering minimal tracking and minimum velocity deviations. Other objects of ACC like the driver comfort, fuel efficiency and car following abilities will be taken as constraints of MPC. Simulations are done using a Laguerre function methods of MPC and is compared with normal MPC method. Simulations are done for different traffic scenarios and the results are compared for the design objectives.
\end{abstract}

Keyword - Adaptive Cruise Control (ACC), Model Predictive Control (MPC), Laguerre function, Transitional maneuvers

\section{INTRODUCTION}

Automobile manufacturers are currently developing cars equipped with Adaptive Cruise Control (ACC) systems which are designed, not only to travel at a set desired speed and keep a safe distance from the preceding vehicle but also accelerated and decelerates according to the state of preceding vehicle. Additionally the car gives improved fuel economy and driver comfort by optimizing the acceleration and breaking of the vehicle.Shaout [1] suggest the improvement in cruisetechnology. The drive objectives of the ACC vehicle is achieved by controlling the acceleration, engine power input and breaking signal effectively. Marsden et al. [2] considered necessity of vehicle adapting to the driver driving characteristics so as to improve the drive comfort.Ioannou [3] suggested the use ACC, to reduce fuel consumption of vehicles.His study also shows the effectiveness of ACC in mixed heavy traffic. Additionaldisturbance created in the response of the system due to frequent cut-in fromadjacent lane and frequent acceleration and deceleration should not cause suddensurprise to the driver. Studies were conducted on ACC to improve its Spacingcontrol, fuel efficiency and ability to individual driver characteristics separately.

J.Zhang [4] proposed the controller for longitudinal vehicle following in heavy trucks. He proposed a simple design using PID for vehicle following which could provide a better performance in microscopic level, design also proposed better fuel economy and in turn reduce pollution. A two loop car model was proposed by M.Persson [5] were an outer loop will make the decisions about the required acceleration based on the relative speed of the cars and the inter distance between the vehicles. Second loop that is the inner loop will control the break and throttle pressures so as to maintain the desired acceleration proposed by the outer loop with minimum overshoots and delay.S. Moon [6] proposed the hierarchical model with an upper and lower level controller design forcollision avoidance and vehicle tracking. The upper level controller will take the decisions based on the sensor inputs on relative velocity, inter distance etc. and estimate a desired acceleration. The lower level controller have the vehicle dynamics which will control the throttle and break to attain the desired acceleration.

This paper proposes an advancement over the previous researches, the paper takes into account multiple objectives like improving the fuel efficiency, provide high level of vehicle safety, improving driver comfort by reducing the jerks to minimum in addition to the basic ACC feature of velocity regulation and vehicle following. These objectives tries to conflict each other for example if we try to improve the fuel efficiency by frequently accelerating and decelerating according to the traffic requirements it will increase the jerk and effect the driver 
comfort. So when we try to improve one factor the other factor will reduce automatically. A control signal needs to be designed that provides an optimal value accounting all the objectives.

Hierarchical control design with an upper level and lower level controller as in Fig 1 is used in this paper also. The lower level controller incorporate the longitudinal dynamics of the vehicle [7]. The controller design controls the breaking pressure and throttle pressure effectively with the help of a switching logic.Lower level controller drives the vehicle in the required speed and maintains the inter distance in the required range according to the desired acceleration command given by the upper level controller. By an effective switching algorithm between brake and throttle the lower level controller brings the relative velocity between the vehicles to zero and distance between the vehicles to required safe range. Lower level controller is incorporating the basic vehicle dynamics controlling the brake and throttle effect separately. Throttle is controlled using a PID controller and brake is controlled using a feedback controller. A switching logic is used to switch between the throttle and break control action [8].

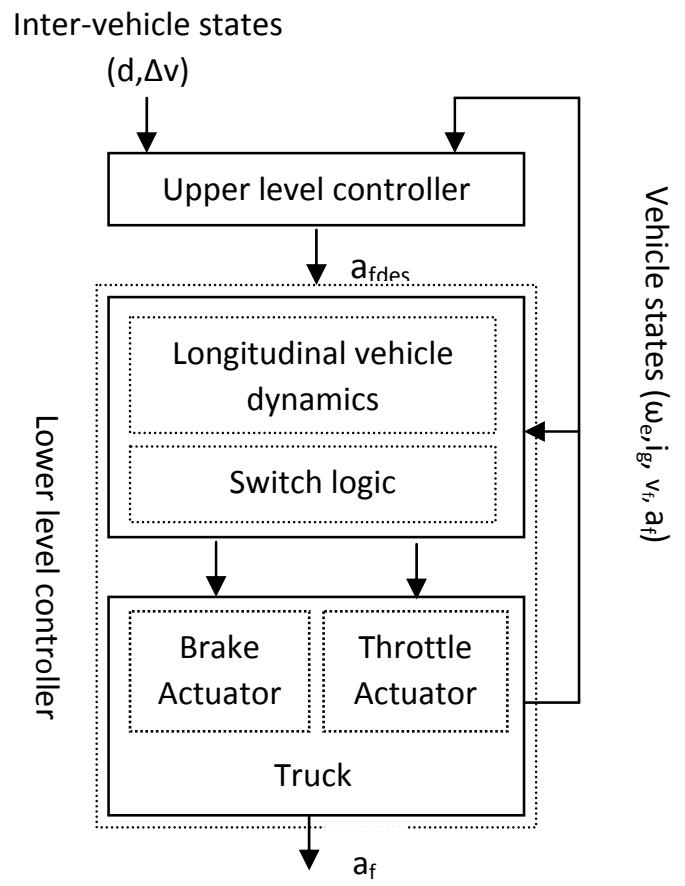

Fig. 1. Hierarchical control architecture

Upper level controller will predict the desired acceleration with which the vehicle should move so as to attain the design objectives. The prediction is done using the vehicle dynamics and the presents value of inter vehicular parameters like relative velocity, variation in inter vehicular distance. A Model predictive control (MPC) can be used effectively to address the issue of designing an ACC system with multiple objectives.V.L. Bageshwar [11] proposes to use the MPC to compute a translational maneuver for vehicle following and spacing control. The paper proposes an optimal control problem where the design objectives are considered as constrains. This paper is extending the use of MPC for addressing the additional objectives like driver comfort also to the constrained optimal control problem. In this Model predictive control the OPC is solved considering the constraints to obtainthe acceleration control signal. Acceleration command signal is computed using receding horizon approach, were the command for next instant is computed using the present values system models and performance index.

The paper uses discrete MPC and system model in discrete domain for the design of the future control trajectory. Moving horizon window predicts the control signal $\mathrm{u}(\mathrm{k})$ and the change in control signal $\Delta \mathrm{u}(\mathrm{k})$ for each instant of time k.The paper uses Laguerre polynomial MPC and the results are compared with traditional method of MPC design. While dealing with a systems like vehicle control where complicated process dynamics, fast sampling and high loop performance is demanded a large number of forward operators will be required. This will lead to very fast and steep changes in the control signal when traditional method of MPC is used. The issue could be overcome by using a soft constraint in the change of control signal $\Delta \mathrm{u}(\mathrm{k})$. The Laguerre polynomial introduces a scaling factor that acts as a constraint to the rate of change of control signal [14]. The objective of driver comfort can be designed more easily by this MPC design. The paper compares the results for traditional MPC and Laguerre function MPC for different traffic scenarios. 


\section{ACC VEHICLE MODEL}

The paper deals with the design and control of the upper level controller alone, which provides a required acceleration command to the lower level controller considering all the kinematics of the vehicle along with the drive requirements and constraints.

Behaviour of the longitudinal vehicle in lower level controller can be represented by a first order equation connecting the acceleration control given by the upper level controller to the velocity acceleration and position of the vehicle. The discrete-time expression of an ACC system represented using difference approximation [9]:

$a(k+1)=\left(1-\frac{T_{s}}{\tau}\right) a(k)+\frac{T_{s}}{\tau} u(k)$

Where Tsand $\mathrm{a}(\mathrm{k})$ represents refers to the sampling period and acceleration at the sampling time $\mathrm{k}$ of the ACC-equipped vehicle respectively.u $(\mathrm{k})$ is the acceleration commend given to lower level controller from upper level controller in the kth instant.

Fig 2 defines a coordinate frame where the state variables of the ACC vehicle is defined with respect to the desired inter vehicular distance $\Delta \operatorname{Sdes}[11]$. The frame is so designed that the origin is located at $\Delta$ Sdes and the frame travels with a velocity equal to that of the target vehicle. The objective could be reframed as reducing the gap $\delta(k)$ and bringing the maneuverof ACC vehicle to the origin.

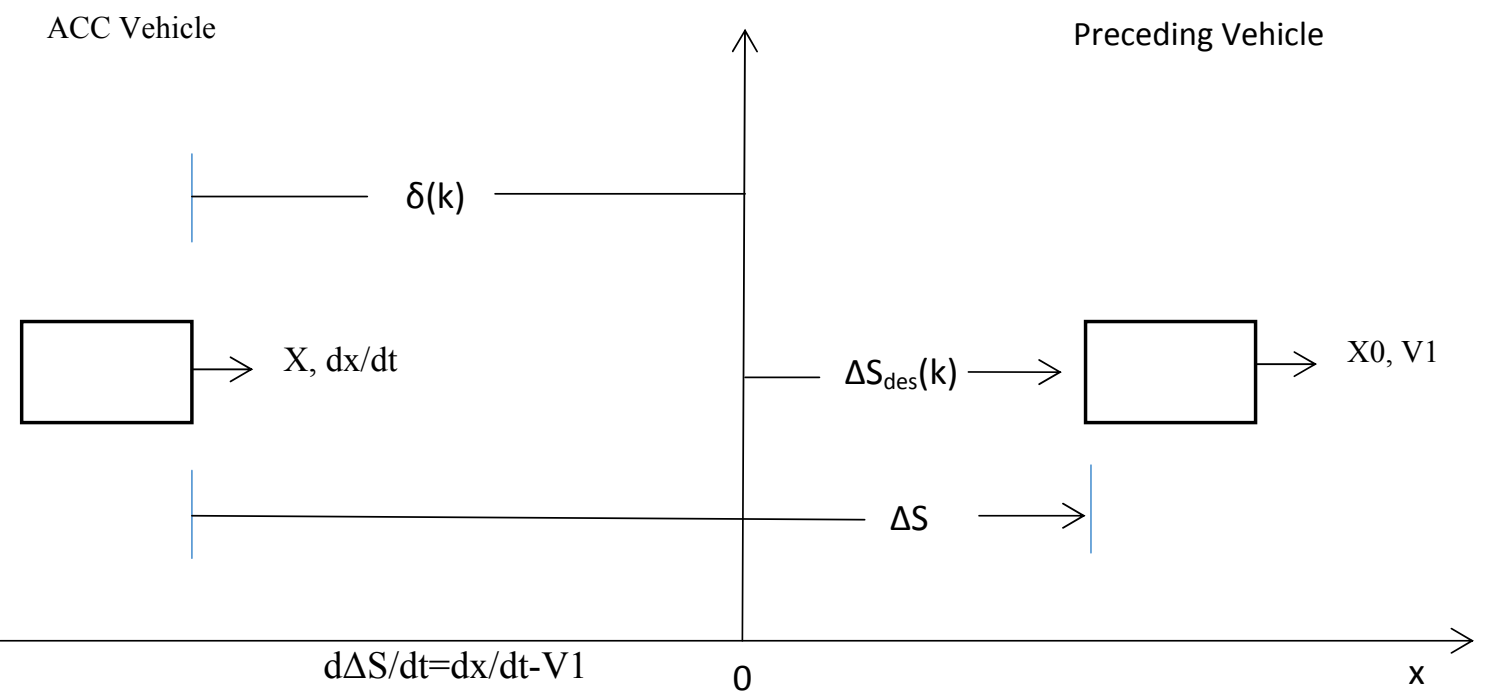

Fig 2 Coordinate frame for transitional maneuvers

The constant time headway (CTH) policy is used:

$\Delta S_{\text {des }}(k)=d_{0}+t_{h} \cdot v(k)$

Where $\Delta S_{\text {des }}(k)$ is the desired spacing between the preceding and ACC equipped vehicle and $\mathrm{v}(\mathrm{k})$ isthe actual velocity of ACC equipped vehicle at sampling time $\mathrm{k}$, th is the desired time headway required to avoid collisionand d0is the fixed safety distance that need to be maintained when the vehicle is at low speed or when vehicle come to stop.

The relative velocity between the vehicles ve and the spacing error $\delta$, are defined as

$\delta(k)=\Delta s(k)-\Delta s_{\text {des }}(k)$

$v_{e}(k)=v_{l}(k)-v(k)$

At sampling time $\mathrm{k}$, the actual distance between the vehicles is $\Delta \mathrm{s}(\mathrm{k})$ and the velocity of the preceding vehicle is represented as $\mathrm{vl}(\mathrm{k})$.

Jerk caused due to the variationsin acceleration is represented as:

$\mathrm{j}(\mathrm{k})=\frac{\mathrm{a}(\mathrm{k})-\mathrm{a}(\mathrm{k}-1)}{\mathrm{T}_{\mathrm{s}}}$

Then the state vector is defined:

$x(k)=\left[\Delta s(k) v(k) v_{e}(k) a(k)\right]^{T}$

Vehicle dynamics of the ACC vehicle and the state of proceeding vehicle is represented using state equations.

$y(k)=\left[\delta(k) v_{e}(k) a(k)\right]^{T}$ 
The system matrices are represented as follows:

$$
A=\left[\begin{array}{cccc}
1 & 0 & T_{s} & -\frac{1}{2} T_{s}^{2} \\
0 & 1 & 0 & T_{s} \\
0 & 0 & 1 & -T_{s} \\
0 & 0 & 0 & 1-\frac{T_{s}}{\tau}
\end{array}\right] B=\left[\begin{array}{c}
0 \\
0 \\
0 \\
\frac{T_{s}}{\tau}
\end{array}\right]
$$

\section{A. Augmented State Space Model}

Applying difference operation on (7) an augmented state space model is obtained as:

$$
\begin{aligned}
& {\left[\begin{array}{c}
\Delta s(k+1) \\
\Delta v(k+1) \\
\Delta v_{e}(k+1) \\
\Delta a(k+1) \\
\delta(k+1) \\
v_{e}(k+1) \\
a(k+1)
\end{array}\right]=\left[\begin{array}{ccccccc}
1 & 0 & T_{s} & -\frac{1}{2} T_{s}^{2} & 0 & 0 & 0 \\
0 & 1 & 0 & T_{s} & 0 & 0 & 0 \\
0 & 0 & 1 & -T_{s} & 0 & 0 & 0 \\
0 & 0 & 0 & \left(1-\frac{T_{s}}{\tau}\right) & 0 & 0 & 0 \\
1 & -t_{h} & T_{s} & -T_{s}\left(\frac{1}{2} T_{s}+t_{h}\right) & 1 & 0 & 0 \\
0 & 0 & 1 & T_{s} & 0 & 1 & 0 \\
0 & 0 & 0 & \left(1-\frac{T_{s}}{\tau}\right) & 0 & 0 & 1 \\
\Delta v(k) \\
\Delta v_{e}(k) \\
\Delta a(k) \\
\delta(k) \\
v_{e}(k) \\
a(k)
\end{array}\right]+\left[\begin{array}{c}
\Delta s(k) \\
\frac{1}{\tau} \\
0 \\
0 \\
T_{s} \\
0 \\
T_{s} \\
\tau
\end{array}\right] \Delta u(k)} \\
& {\left[\begin{array}{c}
\delta(k) \\
v_{e}(k)
\end{array}\right]=\left[\begin{array}{lllllll}
0 & 0 & 0 & 0 & 1 & 0 & 0 \\
0 & 0 & 0 & 0 & 0 & 1 & 0 \\
0 & 0 & 0 & 0 & 0 & 0 & 1
\end{array}\right]\left[\begin{array}{c}
0 \\
\Delta s(k) \\
\Delta v(k) \\
\Delta v_{e}(k) \\
\Delta a(k) \\
\delta(k) \\
v_{e}(k) \\
a(k)
\end{array}\right]}
\end{aligned}
$$

Augmented model eq (8) is used for the future predictions of system states and control signals in MPC solution. The objective of the paper is to design an ACC equipped vehicle that can automatically regulate its speed according to the variations in speed of the preceding vehicle, vl, and to maintain the distance between the two vehicles to the desired values, $\Delta$ sdes:

$$
\text { Objectives: } \quad v_{e}(k) \rightarrow 0 \text { and } \delta(k) \rightarrow 0 \text {, as } k \rightarrow \text { inf }
$$

$\delta$ is the spacing error and ve is the relative velocity,

\section{B. Constraints}

Passenger comfort objective is met by keeping the absolute value of acceleration and jerk as small as possible.

Objective: $\left\{\begin{array}{l}\min |a(k)| \\ \min |j(k)|\end{array}\right.$

To avoid collision a distance larger than the critical minimum safe distance dc should be maintained with the preceding vehicle.

Hard constraint: $\Delta s(k) \geq d_{c}$

Maximum and minimum values are also defines as constrains for Velocity, acceleration, jerk and control command:

Hard constraint: $v_{\min } \leq v(k) \leq v_{\max }$

Hard constraint: $a_{\min } \leq a(k) \leq a_{\max }$

Hard constraint: $u_{\min } \leq u(k) \leq u_{\max }$

Hard constraint: $\Delta u_{\min } \leq \Delta u(k) \leq \Delta u_{\max }$

Hard constraint: $j_{\min } \leq j(k) \leq j_{\max }$

In matrix form the constraints Eq. (11) can be written as: 
s.t $\left\{\begin{array}{c}M \leq L x(k+m) \leq N \\ u_{\min } \leq u(k+m) \leq u_{\max } \\ \Delta u_{\min } \leq \Delta u(k+m) \leq \Delta u_{\max }\end{array}\right.$

Where

$M=\left[\begin{array}{c}d_{c} \\ v_{\min } \\ a_{\min }\end{array}\right] N=\left[\begin{array}{c}i n f \\ v_{\max } \\ a_{\max }\end{array}\right] L=\left[\begin{array}{llll}1 & 0 & 0 & 0 \\ 0 & 1 & 0 & 0 \\ 0 & 0 & 0 & 1\end{array}\right]$

Substituting (4.5) to (12) the constraints are rewritten as

$\Omega \Delta U(k+m) \leq T$

Where

$$
\begin{aligned}
& \Omega=\left[\begin{array}{llllll}
L B & -L B & I & -I & I & -I
\end{array}\right]^{T} \\
& T=\left[\begin{array}{c}
N-L A x(k) \\
-M+L A x(k) \\
\Delta u_{\max } \\
-\Delta u_{\min } \\
u_{\max }-u(k-1) \\
-u_{\min }+u(k-1)
\end{array}\right]
\end{aligned}
$$

The objective of upper level controller is to find an optimum value of $\Delta U$ that minimizes the cost function

$$
J=\sum_{m=1}^{N_{p}} x\left(k_{i}+m \mid k_{i}\right)^{T} Q x\left(k_{i}+m \mid k_{i}\right)^{T}+\eta \Delta U^{T} R_{L} \Delta U
$$

Constrained to $\Omega \Delta U(k+m) \leq T$

The objective is achieved by both traditional and Laguerre function method of MPC.

\section{SIMULATION OF LAGUERRE MPC CONTROLLER}

ACC algorithm using traditional MPC controller and Laguerre function method of MPC controllers are simulated and compared. The objectives are assessed from the simulation results. The prime objective is safety which is measured on the basis of how effectively collision is avoided which is in turn the ability to maintain a safe inter vehicular distance. Regulating the velocity and inter vehicular distance to the desired values, measure of car following ability. The magnitude of acceleration and jerk gives an account of drive comfort. Design algorithm is simulated for different traffic conditions like preceding vehicle with varying speed, cutting in, cutting out, stationary and hard stop [9].

The parameters values taken for simulation for the ACC equipped vehicle is taken asin table 1:

TABLE 1. Parameters of MPC controller

\begin{tabular}{|c|c|c|}
\hline Parameter & Meaning & Value \\
\hline $\mathrm{T}_{\mathrm{s}}$ & Sampling period & $0.2 \mathrm{sec}$ \\
\hline $\mathrm{t}_{\mathrm{h}}$ & Time headway & $3 \mathrm{sec}$ \\
\hline $\mathrm{T}$ & Time lag & $0.5 \mathrm{sec}$ \\
\hline $\mathrm{d}_{0}$ & Minimum safety distance & $7 \mathrm{~m}$ \\
\hline $\mathrm{d}_{\mathrm{c}}$ & Critical minimum safe distance & $5 \mathrm{~m}$ \\
\hline $\mathrm{v}_{\min }$ & Minimum velocity & $0 \mathrm{~m} / \mathrm{s}$ \\
\hline $\mathrm{v}_{\max }$ & Maximum velocity & $36 \mathrm{~m} / \mathrm{s}$ \\
\hline $\mathrm{a}_{\min }$ & Minimum acceleration & $-5 \mathrm{~m} / \mathrm{s}^{2}$ \\
\hline $\mathrm{a}_{\max }$ & Maximum acceleration & $5 \mathrm{~m} / \mathrm{s}^{2}$ \\
\hline $\mathrm{u}_{\min }$ & Minimum control & $-2.5 \mathrm{~m} / \mathrm{s}^{2}$ \\
\hline $\mathrm{u}_{\max }$ & Maximum control & $5 \mathrm{~m} / \mathrm{s}^{2}$ \\
\hline$\Delta \mathrm{u}_{\min }$ & Minimum control increment & $-2.5 \mathrm{~m} / \mathrm{s}^{2}$ \\
\hline$\Delta \mathrm{u}_{\max }$ & Maximum control increment & $5 \mathrm{~m} / \mathrm{s}^{2}$ \\
\hline $\mathrm{Jmax}$ & Maximum jerk & $2 \mathrm{~m} / \mathrm{s}^{3}$ \\
\hline $\mathrm{Jmin}_{\min }$ & Minimum jerk & $2 \mathrm{~m} / \mathrm{s}^{3}$ \\
\hline $\mathrm{N}_{\mathrm{p}}$ & Prediction horizon & 16 \\
\hline
\end{tabular}




\begin{tabular}{|c|c|c|}
\hline $\mathrm{N}_{\mathrm{c}}$ & Control horizon & 5 \\
\hline $\mathrm{A}$ & Laguerre scaling factor & 0.8 \\
\hline $\mathrm{N}$ & Laguerre parameter & 3 \\
\hline
\end{tabular}

\section{A. Scenario 1: Following a Vehicle With Varying Speed}

Consider the scenario whenthe vehicle preceding our ACC-equipped vehicle is varying its speed. The initial conditions may be taken as the distance between the preceding and ACC vehicle is $50 \mathrm{~m}$, the initial speed of preceding vehicle is $15 \mathrm{~m} / \mathrm{s}$ and ACC-equipped vehicle is travelling at a speed of $10 \mathrm{~m} / \mathrm{s}$, keeping a $5 \mathrm{~m} / \mathrm{s}$ for relative velocity[9]. Constraints are applied on input variation $\Delta \mathrm{u}$, input $\mathrm{u}$, acceleration a, jerk $\mathrm{j}$. Vehicle control is simulated using a traditional MPC and using Laguerre function MPC. Waveform (Fig 3) shows the comparison in inter vehicular distance, Vehicle speed, acceleration and jerk. Fig 4 shows the comparison of the control signal and change in control signal.

Driver comfort and fuel economy is estimated by the magnitude of constraint applied on acceleration, jerk, control input, and incremental change in control. Table 2 shows the maximum constraints that can be applied on traditional and Laguerre function MPC beyond which effective car following is not possible. Simulation results shows that in traditional MPC constrains cannot be minimised to the required limits. Beyond this the system goes to unstable situations. Laguerre function method shows better results as the aim was to minimise the constraints as minimum as possible also shows good vehicle following property.
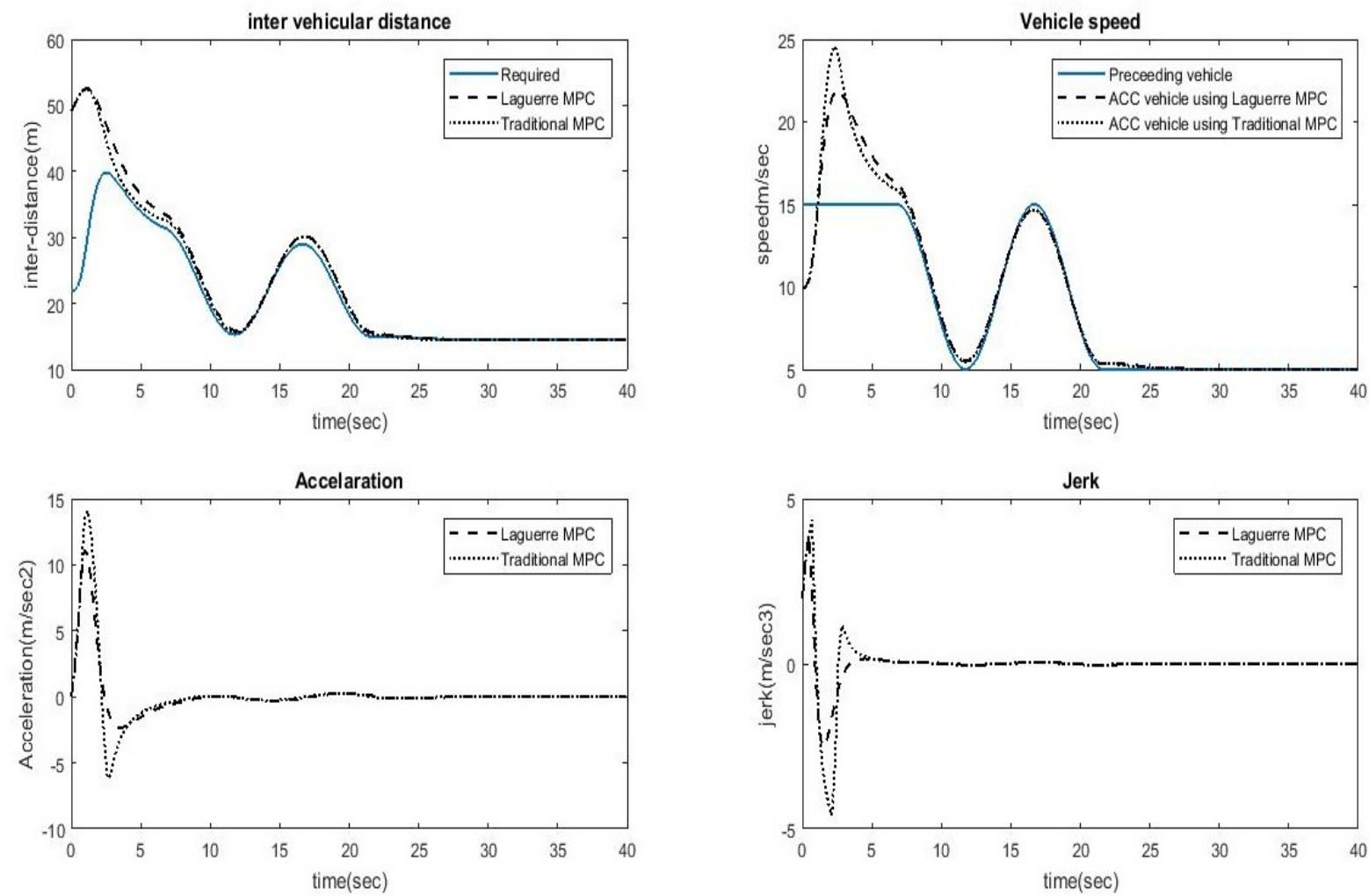

Fig: 3Response of ACC vehicle using Traditional MPC and Laguerre function MPC controller for varying preceding vehicle speed. 

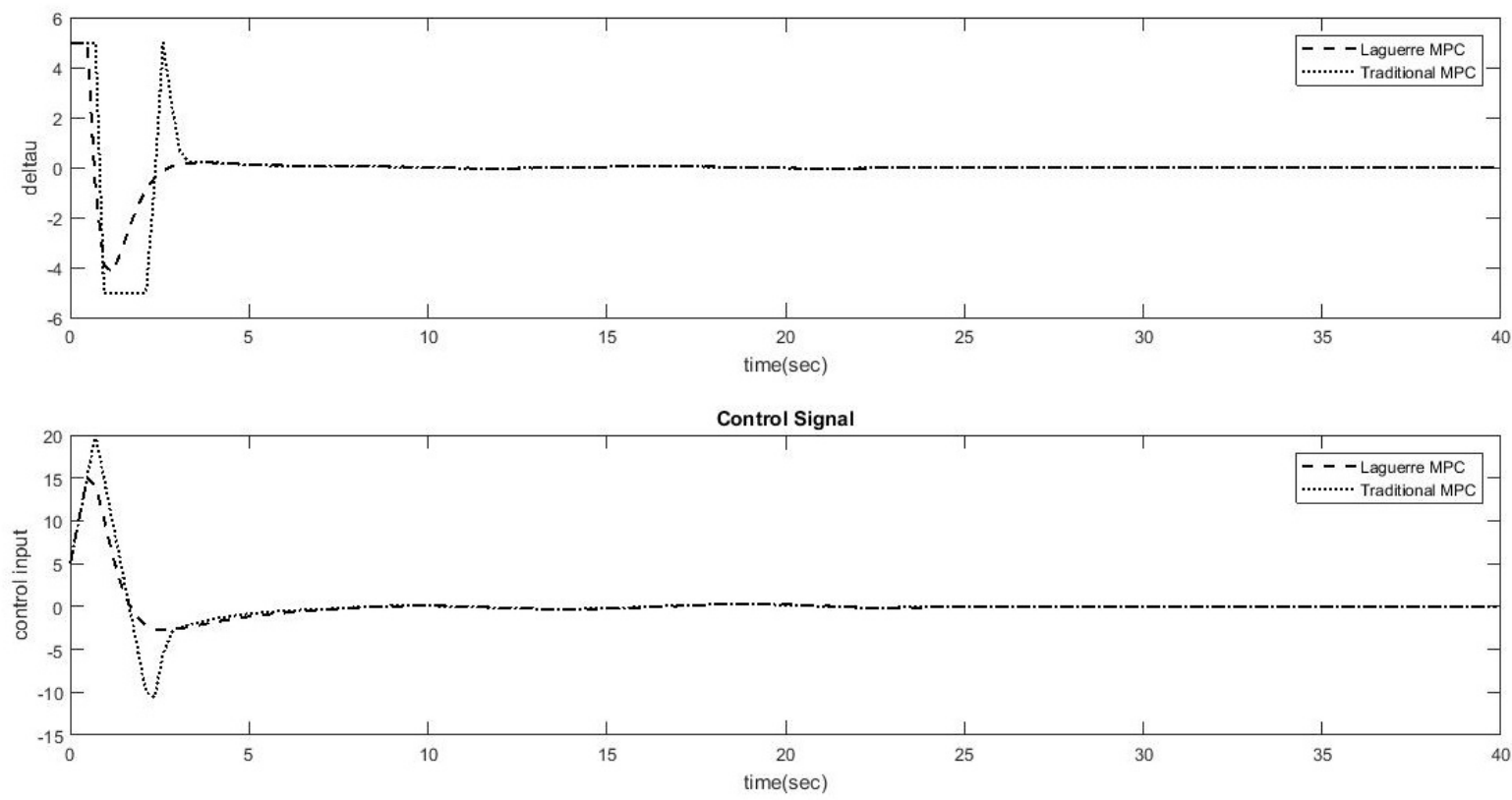

Fig: 4Control signal for Traditional MPC and Laguerre function MPC controller for varying preceding vehicle speed.

\section{B. Scenario 2: A Vehicle Cutting In From Adjacent Lane}

The scenario in which a vehicle cuts in before the ACC equipped vehicle from the adjacent lane with a velocity of $10 \mathrm{~m} / \mathrm{s}$ when the velocity of ACC equipped vehicle was $15 \mathrm{~m} / \mathrm{s}(-5 \mathrm{~m} / \mathrm{s}$ for relative velocity) and the distance between the vehicle suddenly changes to $15 \mathrm{~m}$ [9]. Waveform (Fig 5) shows the comparison in inter vehicular distance, Vehicle speed, acceleration and jerk. Fig 6 shows the comparison of the control signal and change in control signal.
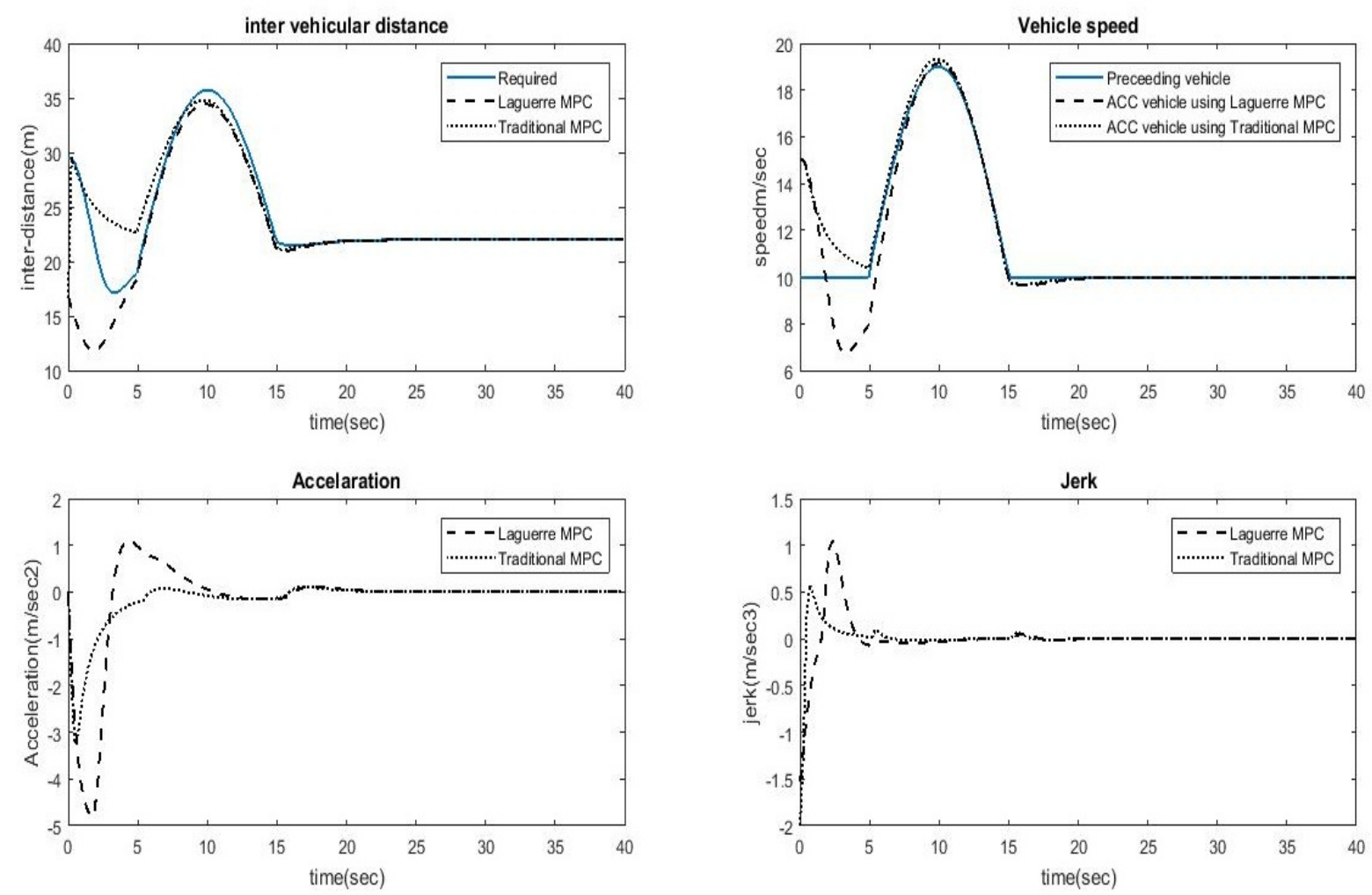

Fig 5Response of ACC vehicle using Traditional MPC and Laguerre function MPC controller when a preceding vehicle cuts in. 

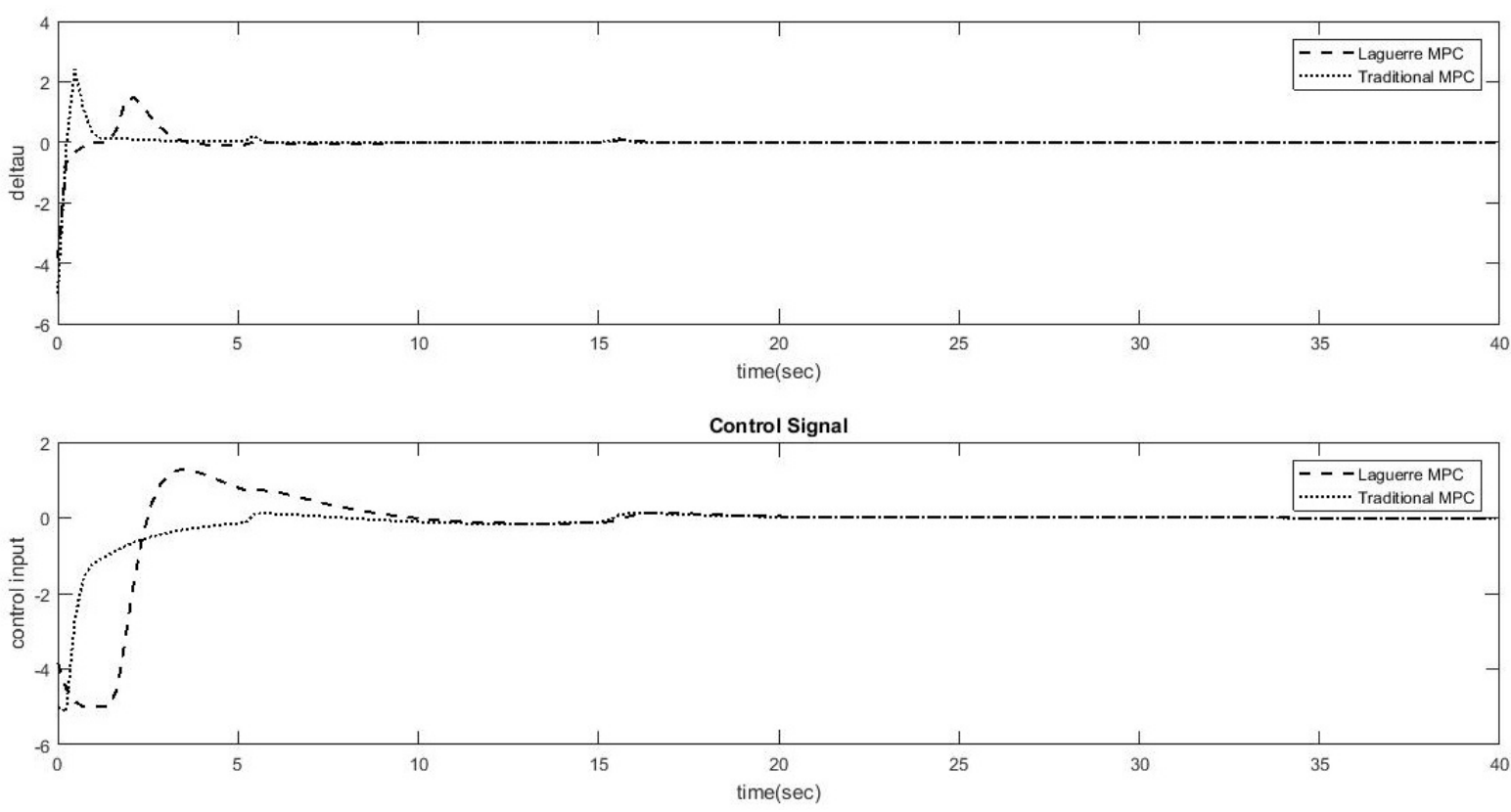

Fig 6Control signal for Traditional MPC and Laguerre function MPC controller when a preceding vehicle cuts in.

\section{Scenario 3: Vehicle Cuts Out to Adjacent Lane}

The scenario where the vehicle which was followed initially suddenly moves out to adjacent lane and a new vehicle comes a preceding vehicle. In the new scenario the velocity of the ACC equipped vehicle and the new preceding vehicle are $10 \mathrm{~m} / \mathrm{s}$ and $20 \mathrm{~m} / \mathrm{s}$, respectively and the distance between the vehicles become $70 \mathrm{~m}$ [9].Waveform (Fig 7) shows the comparison in inter vehicular distance, Vehicle speed, acceleration and jerk. Fig 8 shows the comparison of the control signal and change in control signal.
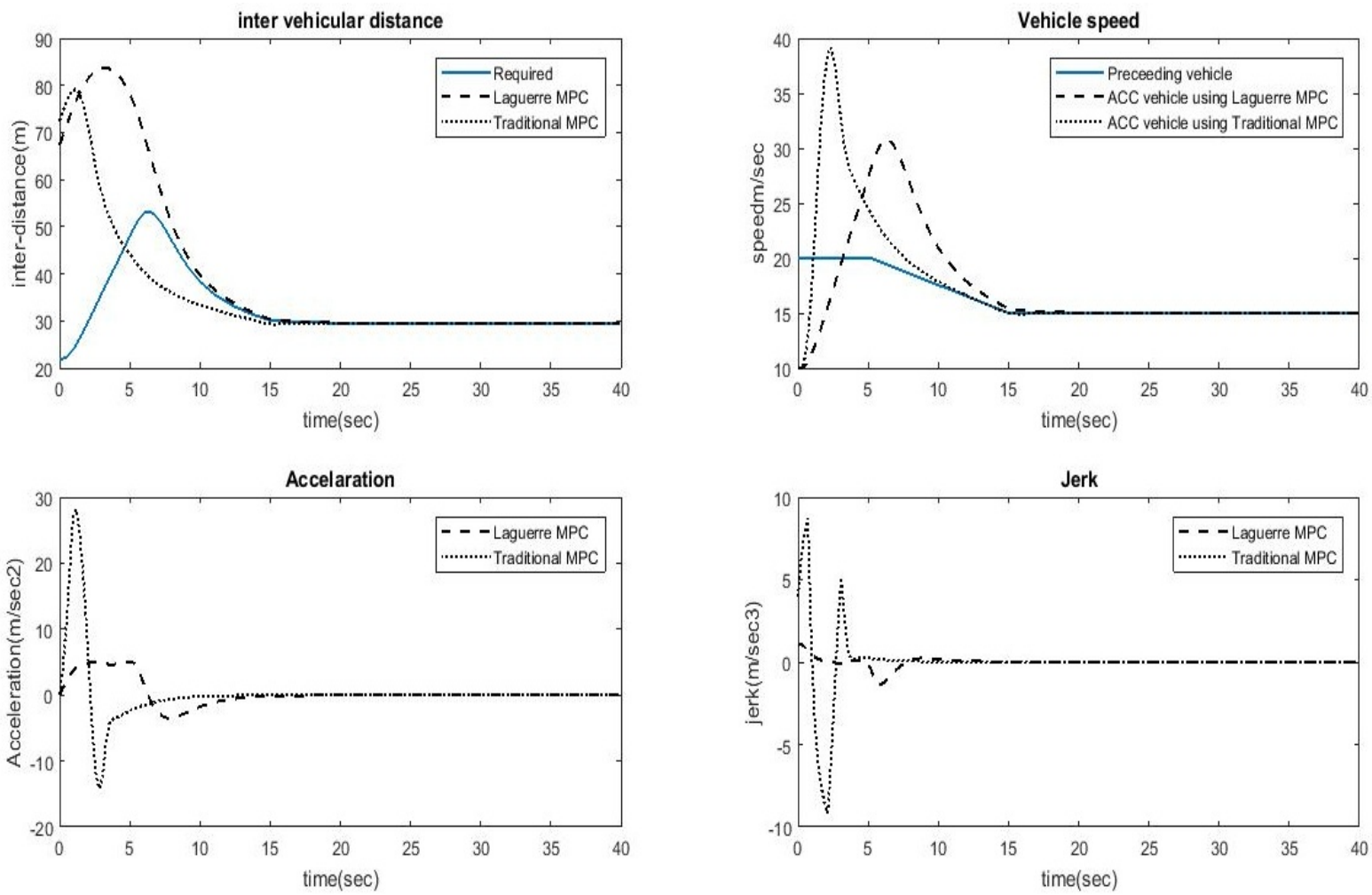

Fig 7 Response of ACC vehicle using Traditional MPC and Laguerre function MPC controller when a preceding vehicle cuts out to adjacent lane. 

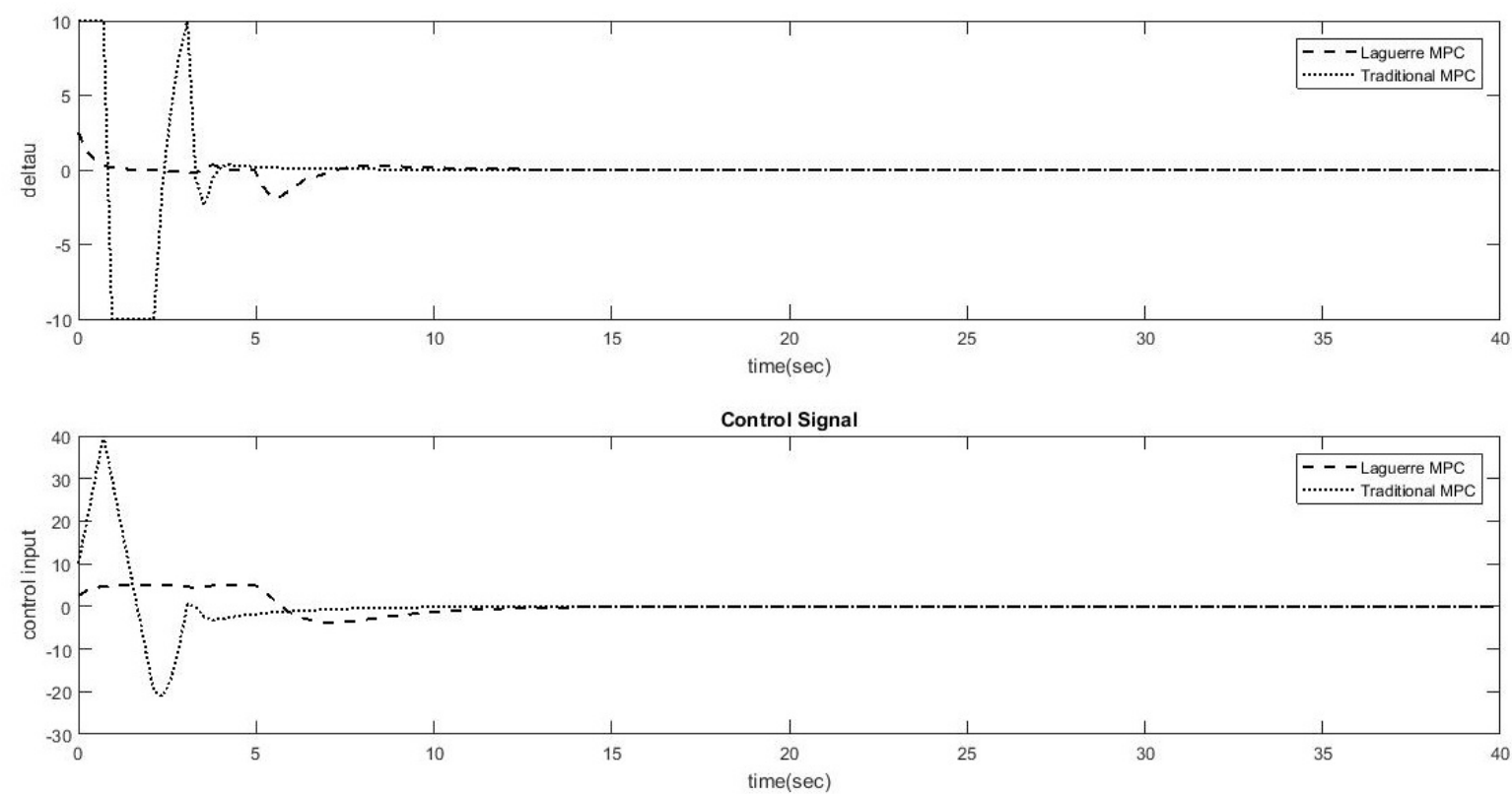

Fig 8Control signal for Traditional MPC and Laguerre function MPC controller when a preceding vehicle cuts out to adjacent lane.

\section{Scenario 4: ACC Vehicle Approaches a Stationary Vehicle.}

Consider the scenario where the ACC-equipped vehicle approaches a stationary vehicle and the initial conditions may be taken as the velocity of ACC equipped vehicle is $10 \mathrm{~m} / \mathrm{s}$ and the distance between the ACC equipped vehicle and the preceding vehicle is $100 \mathrm{~m}$. ACC vehicle have to come to stop to avoid collision and a minimum stopping distance have to be kept between the vehicles when it stop.Waveform (Fig 9) shows thecomparison in inter vehicular distance, Vehicle speed, acceleration and jerk. Fig 10 shows the comparison of the control signal and change in control signal.
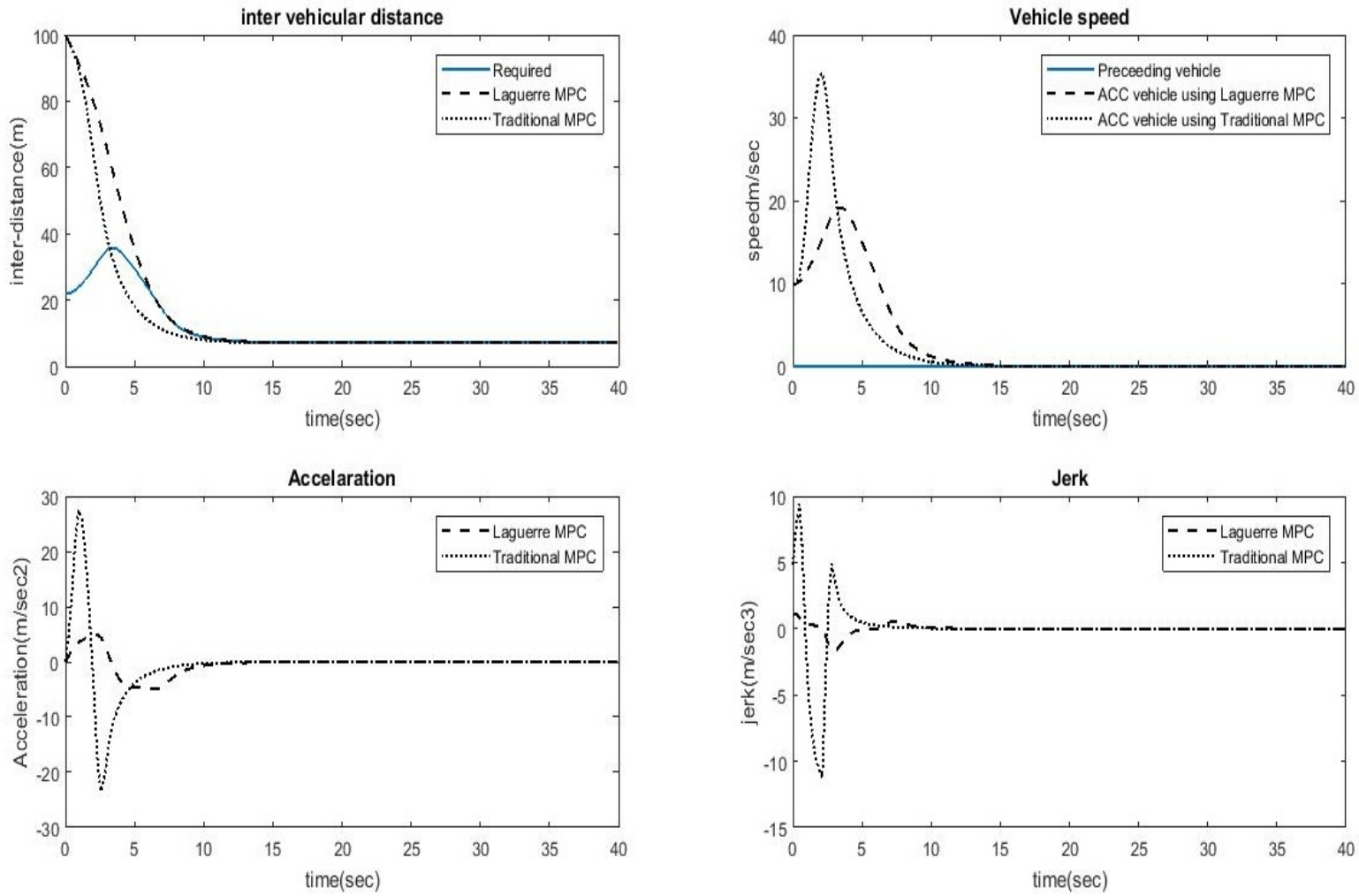

Fig: 9Response of ACC vehicle using Traditional MPC and Laguerre function MPC controller when a preceding vehicle is stationary. 

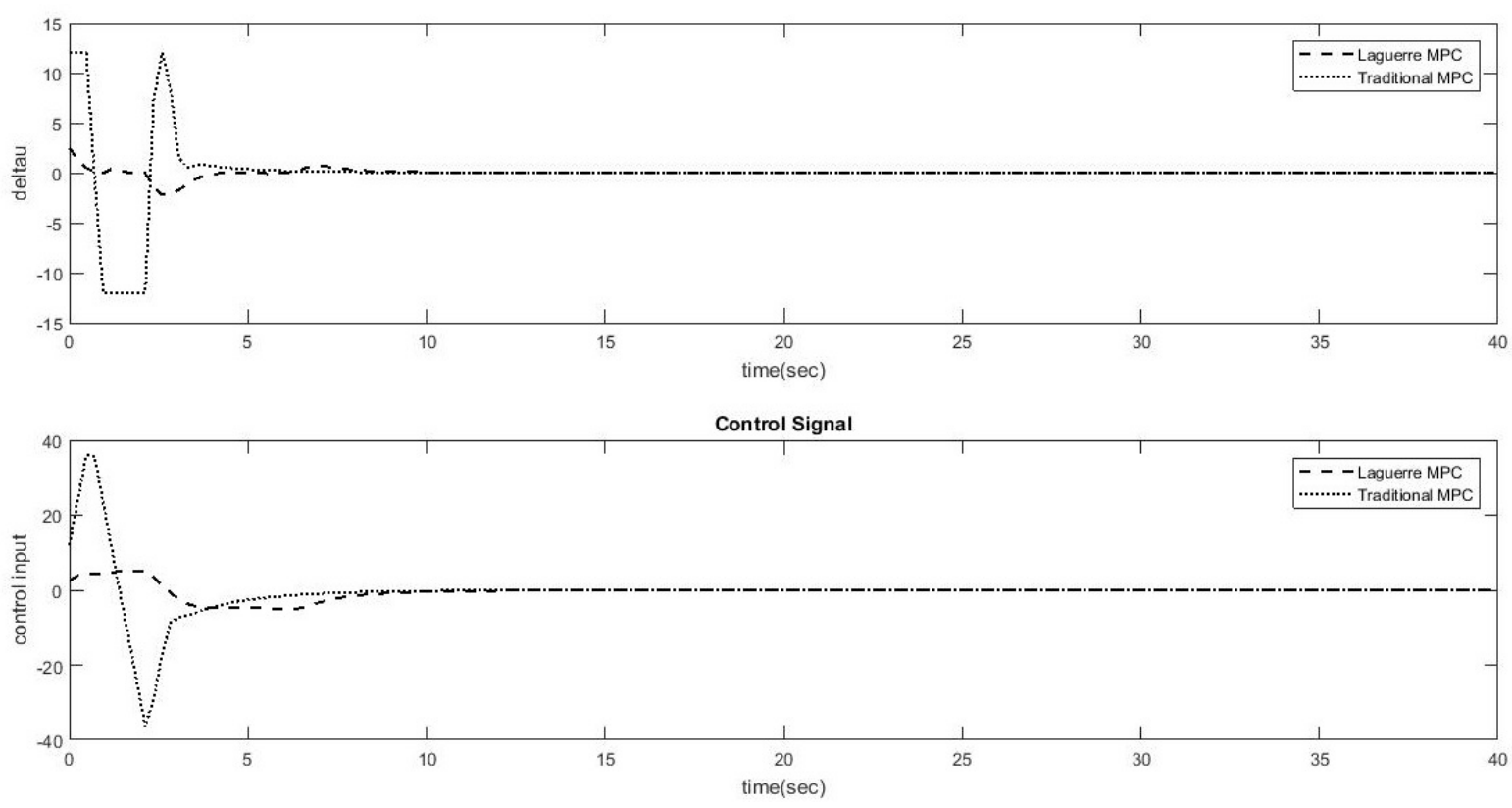

Fig: 10Control signal for Traditional MPC and Laguerre function MPC controller when a preceding vehicle is stationary.

\section{E. Scenario 5: Preceding Vehicle Performs a Hard Stop.}

Scenario when the preceding vehicle stops suddenly, initial condition is taken as the velocity of ACC equipped vehicle is $20 \mathrm{~m} / \mathrm{s}$ and the distance between the ACC equipped vehicle and the preceding vehicle is $50 \mathrm{~m}$. [9]. Waveform (Fig 11) shows the comparison in inter vehicular distance, Vehicle speed, acceleration and jerk. Fig 12 shows the comparison of the control signal and change in control signal.
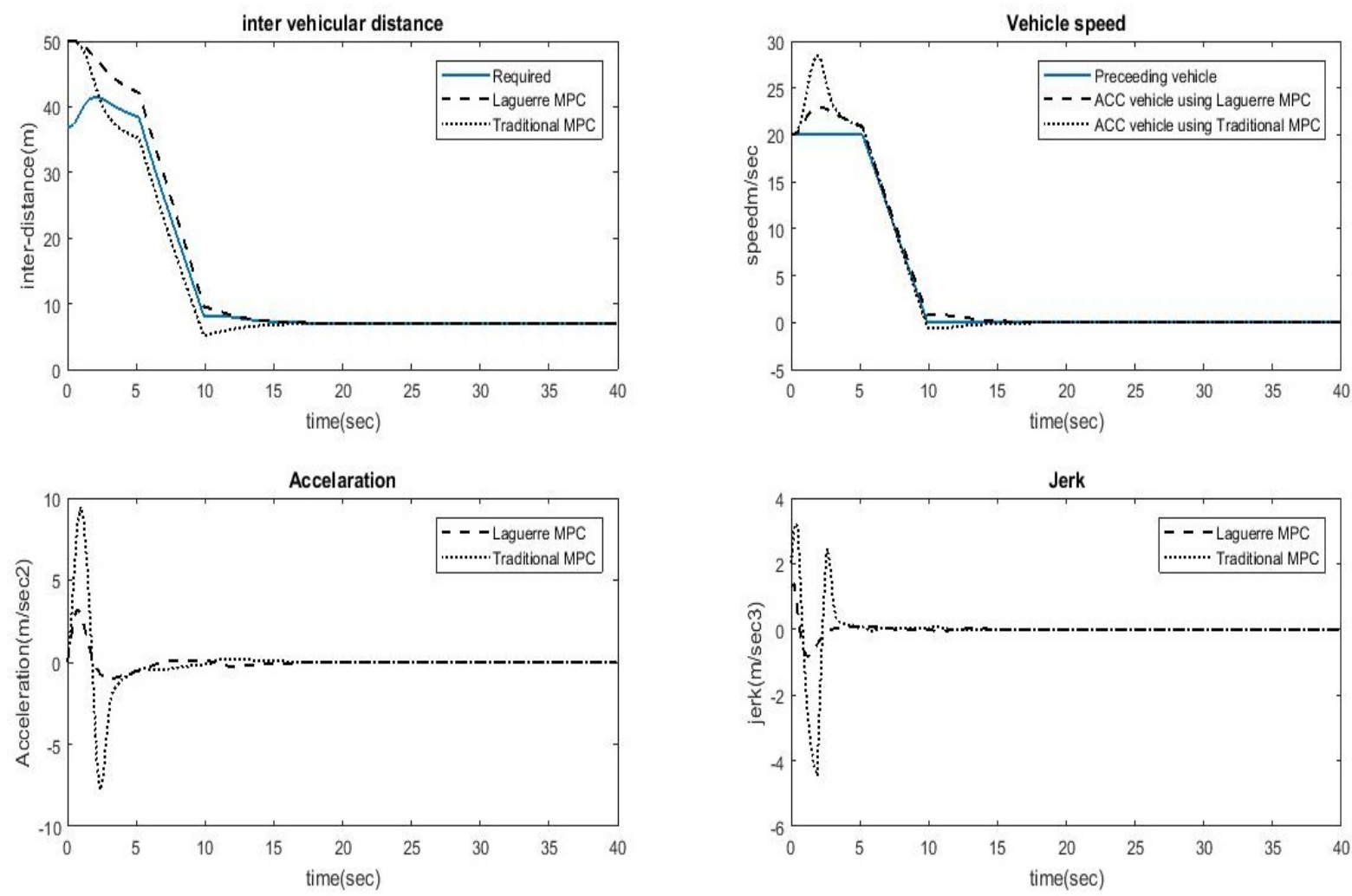

Fig 11Response of ACC vehicle using Traditional MPC and Laguerre function MPC controller when a preceding vehicle stops suddenly. 

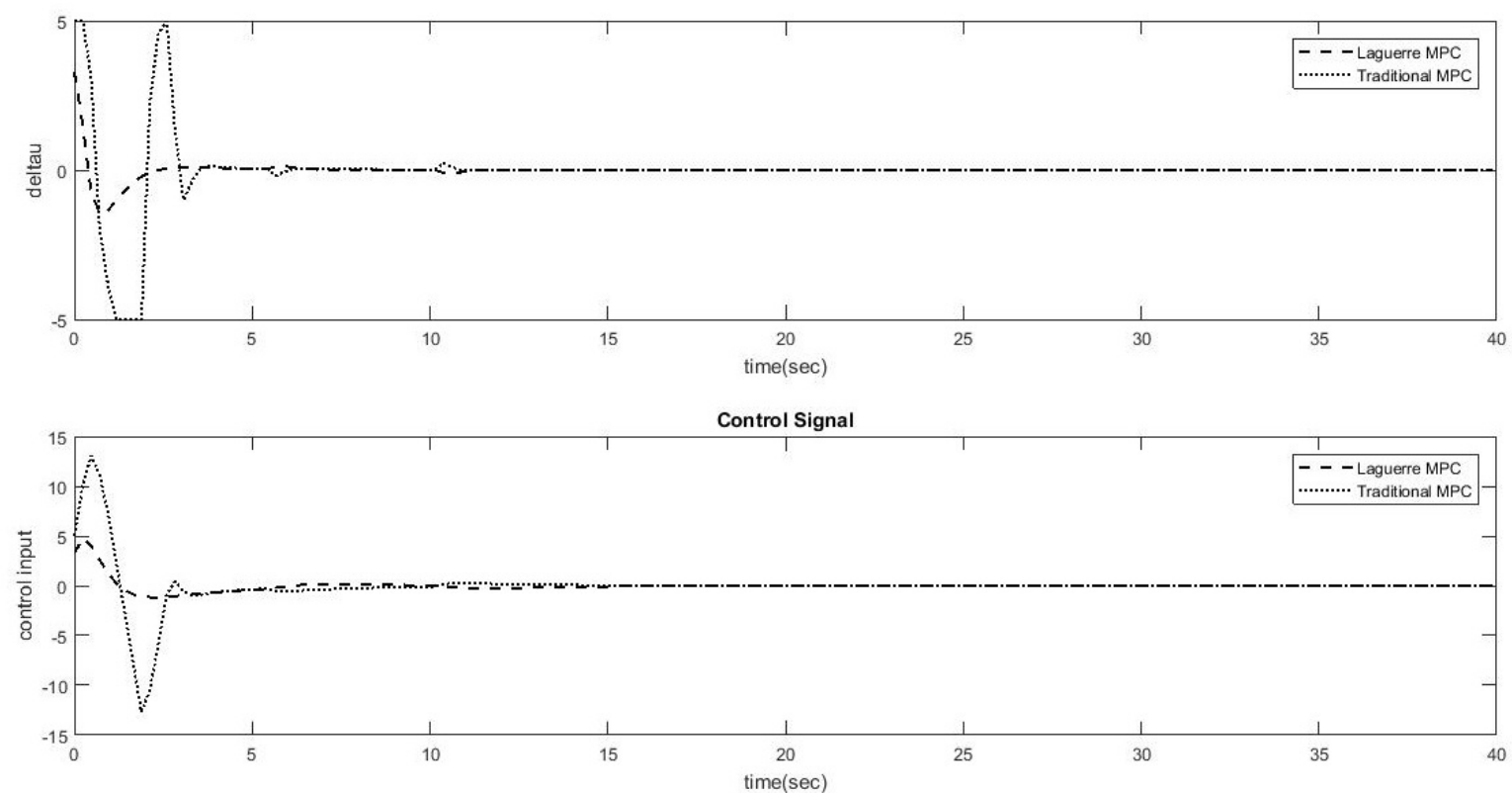

Fig: 12 Control signal for Traditional MPC and Laguerre function MPC controller when a preceding vehicle stops suddenly.

Laguerre function method of MPC show better results with for smaller constrain range compared to the traditional method of MPC. Fuel efficiency and driver comfort is more for Laguerre function method as it is measured by the magnitude of acceleration and jerk. Fig 12 shows the $\mathrm{u}$ and $\Delta \mathrm{u}$ plot for varying speed scenario where in the constraints limits are increased to $-15 \leq \mathrm{a} \leq 15,-5 \leq \mathrm{J} \leq 5,-25 \leq \mathrm{u} \leq 25,-5 \leq \Delta \mathrm{u} \leq 5$. Figure shows that Laguerre function method gives a smooth control signal where as traditional method have sudden changes in $\Delta \mathrm{u}$ values. Settling time for the $\Delta \mathrm{u}$ signals is small for Laguerre function method compared to traditional method.

TABLE 2. Analysis Table On Constrains For Different Scenarios

\begin{tabular}{|c|c|c|c|c|c|c|c|c|c|c|c|}
\hline & \multirow{2}{*}{ 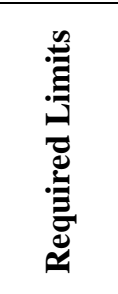 } & \multicolumn{2}{|c|}{ Varying speed } & \multicolumn{2}{|c|}{ Vehicle cuts in } & \multicolumn{2}{|c|}{ Vehicle cuts out } & \multicolumn{2}{|c|}{$\begin{array}{c}\text { Vehicle } \\
\text { stationary }\end{array}$} & \multicolumn{2}{|c|}{ Hard stop } \\
\hline & & 吾 & 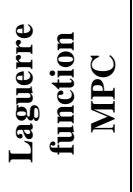 & 吾 & 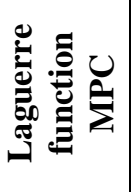 & 预 & 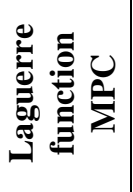 & 预 U & 记 & 预 & 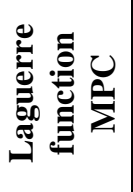 \\
\hline $\begin{array}{c}\text { Inter- } \\
\text { Vehicula } \\
\mathbf{r} \\
\text { distance } \\
\end{array}$ & $\begin{array}{c}5 \leq \Delta \mathrm{S} \\
\leq \inf \end{array}$ & $\begin{array}{c}14.42 \\
\leq \Delta \mathrm{S} \leq \\
52.50\end{array}$ & $\begin{array}{c}14.50 \\
\leq \Delta \mathrm{S} \leq \\
54.54\end{array}$ & $\begin{array}{c}11.81 \\
\leq \Delta \mathrm{S} \leq \\
32.42\end{array}$ & $\begin{array}{c}16.60 \\
\leq \Delta \mathrm{S} \leq \\
34.79\end{array}$ & $\begin{array}{c}29.28 \\
\leq \Delta \mathrm{S} \leq \\
79.17\end{array}$ & $\begin{array}{c}20.50 \\
\leq \Delta \mathrm{S} \leq \\
83.75\end{array}$ & $\begin{array}{l}7 \leq \Delta S \\
\leq 100\end{array}$ & $\begin{array}{l}7 \leq \Delta \mathrm{S} \\
\leq 100\end{array}$ & $\begin{array}{c}7 \leq \Delta \mathrm{S} \\
\leq 50\end{array}$ & $\begin{array}{c}7 \leq \Delta \mathrm{S} \\
\leq 50\end{array}$ \\
\hline Velocity & $\begin{array}{l}0 \leq \mathrm{V} \\
\leq 36\end{array}$ & $\begin{array}{c}5.00 \leq \\
V \leq \\
24.57\end{array}$ & $\begin{array}{c}5.00 \leq \\
V \leq \\
21.80\end{array}$ & $\begin{array}{c}6.75 \leq \\
V \leq \\
19.15\end{array}$ & $\begin{array}{c}9.67 \leq \\
V \leq \\
19.33\end{array}$ & $\begin{array}{c}10 \leq \mathrm{V} \\
\leq \\
39.15\end{array}$ & $\begin{array}{c}10 \leq \mathrm{V} \\
\leq \\
30.76\end{array}$ & $\begin{array}{c}0 \leq \mathrm{V} \\
\leq \\
35.34\end{array}$ & $\begin{array}{c}0 \leq \mathrm{V} \leq \\
19.17\end{array}$ & $\begin{array}{c}0 \leq \mathrm{V} \\
\leq \\
28.44\end{array}$ & $\begin{array}{c}0 \leq \mathrm{V} \leq \\
22.95\end{array}$ \\
\hline $\begin{array}{c}\text { Accelera } \\
\text { tion }\end{array}$ & $\begin{array}{c}-5 \leq \mathrm{a} \\
\leq 5\end{array}$ & $\begin{array}{c}-6.13 \leq \\
a \leq \\
14.08\end{array}$ & $\begin{array}{c}-2.26 \leq \\
\mathrm{a} \leq \\
4.96\end{array}$ & $\begin{array}{c}-4.81 \leq \\
\mathrm{a} \leq \\
1.08\end{array}$ & $\begin{array}{c}-3.24 \leq \\
\mathrm{a} \leq \\
0.10\end{array}$ & $\begin{array}{l}-14.24 \\
\leq \mathrm{a} \leq \\
28.16\end{array}$ & $\begin{array}{c}-3.50 \leq \\
\mathrm{a} \leq \\
4.97\end{array}$ & $\begin{array}{l}-23.43 \\
\leq \mathrm{a} \leq \\
27.37\end{array}$ & $\begin{array}{c}-4.97 \leq \\
\mathrm{a} \leq \\
4.89\end{array}$ & $\begin{array}{c}-7.79 \leq \\
\mathrm{a} \leq \\
9.46\end{array}$ & $\begin{array}{c}-0.99 \leq \\
\mathrm{a} \leq \\
3.22\end{array}$ \\
\hline Jerk & $\begin{array}{l}-2 \leq \mathrm{J} \\
\leq 2\end{array}$ & $\begin{array}{c}-4.56 \leq \\
\mathrm{J} \leq \\
4.35\end{array}$ & $\begin{array}{c}-1.32 \leq \\
\mathrm{J} \leq \\
1.12 \\
\end{array}$ & $\begin{array}{c}-1.53 \leq \\
\mathrm{J} \leq \\
1.05 \\
\end{array}$ & $\begin{array}{l}-2.0 \leq \mathrm{J} \\
\leq 0.57\end{array}$ & $\begin{array}{c}-9.12 \leq \\
\mathrm{J} \leq \\
8.70\end{array}$ & $\begin{array}{c}-1.36 \leq \\
\mathrm{J} \leq \\
1.10 \\
\end{array}$ & $\begin{array}{c}-11.1 \leq \\
\mathrm{J} \leq \\
9.40\end{array}$ & $\begin{array}{c}-1.63 \leq \\
\mathrm{J} \leq \\
1.12 \\
\end{array}$ & $\begin{array}{c}-4.46 \leq \\
\mathrm{J} \leq \\
3.20 \\
\end{array}$ & $\begin{array}{c}-0.82 \leq \\
\mathrm{J} \leq \\
1.38 \\
\end{array}$ \\
\hline $\begin{array}{c}\text { Control } \\
\text { input }\end{array}$ & $\begin{array}{c}-5 \leq \mathrm{u} \\
\leq 5\end{array}$ & $\begin{array}{c}-10.59 \\
\leq \mathrm{u} \leq \\
20\end{array}$ & $\begin{array}{c}-2.59 \leq \\
\mathrm{u} \leq 5\end{array}$ & $\begin{array}{c}-5.0 \leq \\
\mathrm{u} \leq \\
1.27\end{array}$ & $\begin{array}{l}-5 \leq \mathrm{u} \\
\leq 0.13\end{array}$ & $\begin{array}{c}-21.12 \\
\leq \mathrm{u} \leq \\
40\end{array}$ & $\begin{array}{c}-3.81 \leq \\
\mathrm{u} \leq 5\end{array}$ & $\begin{array}{c}-36.39 \\
\leq \mathrm{u} \leq \\
36\end{array}$ & $\begin{array}{c}-5 \leq \mathrm{u} \\
\leq 5\end{array}$ & $\begin{array}{l}-12.72 \\
\leq \mathrm{u} \leq \\
13.06\end{array}$ & $\begin{array}{c}-1.15 \leq \\
\mathrm{u} \leq \\
4.77\end{array}$ \\
\hline $\begin{array}{c}\begin{array}{c}\text { Rate of } \\
\text { Change } \\
\text { of } \\
\text { control } \\
\text { input }\end{array} \\
\end{array}$ & $\begin{array}{c}-2 \leq \\
\Delta \mathrm{u} \leq 5\end{array}$ & $\begin{array}{c}-5.0 \leq \\
\Delta \mathrm{u} \leq 5\end{array}$ & $\begin{array}{c}-1.86 \leq \\
\Delta \mathrm{u} \leq \\
2.73\end{array}$ & $\begin{array}{c}-3.82 \leq \\
\Delta \mathrm{u} \leq \\
1.46\end{array}$ & $\begin{array}{c}-5.0 \leq \\
\Delta \mathrm{u} \leq \\
2.41\end{array}$ & $\begin{array}{c}-10 \leq \\
\Delta \mathrm{u} \leq \\
10\end{array}$ & $\begin{array}{c}-1.82 \leq \\
\Delta \mathrm{u} \leq \\
2.50\end{array}$ & $\begin{array}{c}-12 \leq \\
\Delta \mathrm{u} \leq \\
12\end{array}$ & $\begin{array}{c}-2.20 \leq \\
\Delta \mathrm{u} \leq \\
2.50\end{array}$ & $\begin{aligned}-5 & \leq \Delta \mathrm{u} \\
& \leq 5\end{aligned}$ & $\begin{array}{c}-1.40 \leq \\
\Delta \mathrm{u} \leq \\
3.27\end{array}$ \\
\hline
\end{tabular}


Laguerre function method of MPC give better results for constrained applications. The controller gives good distance and velocity tracking keeping the acceleration and jerk to the required limits (Table 2). Traditional method of MPC shows sudden variations in $\Delta \mathrm{u}$ and $\mathrm{u}$, whereas Laguerre method shows smooth variations in $\Delta \mathrm{u}$ and $\mathrm{u}$ (fig 12). Comparing the result for all the 5 scenarios it's clear that Laguerre function method of MPC gives better results compared to traditional method of MPC.

\section{CONCLUSION}

The paper designs an adaptive cruise vehicle that comprehensivelyaddresses the objectives of following the preceding vehicle with desired speed and safe distance between the vehicles, fuel economy, and driver comfort. A hierarchical control architecture was used for the vehicle design. Lowerlevel controller was designed with a switching logic to switch between throttlecontrol by a PID controller and brake control using a feedback controller. Adaptive cruise control algorithm using Laguerre function method of MPCwas used to satisfy multiobjectives. Tracking error and vehicle followingrequirements were taken as the cost function and driver comfort, fuel-economy,safety was taken as constraints. Simulation graphs for all the five different traffic scenarios shows that the performances of the designed ACC algorithm avoiding collision effectively satisfying the criterion of safety. Velocity and inter vehicular distance is controlled and regulated effectively satisfying the objective of car-following. Magnitude variations in acceleration and jerk estimates the driving comfort.Result shows that the designed Laguerre function type of ACC algorithmmeets safety and car-following objective and also improves driving comfort andfuel efficiency compared to the traditional MPC control. The use of advanced control algorithm will encourage the vehicle manufactures to implement ACC control to more commercial vehicle as this technology will bring not only commercial benefits to the users about also will improve the safety in driving and reduce the stress and discomforts of driving. The research can be improved in future by adding more nonlinear dynamics of vehicle into the MPC design.

\section{REFERENCES}

[1] Shaout, and M. A. Jarrahz, "Cruise Control Technology Review.” Computers Elect. Engg Vol. 23. No. 4, pp. $259-271.1997$.

[2] G. Marsden, M. McDonald, and M. Brackstone, “Towards an understanding ofadaptive cruise control," Transport. Res. Pt. C, pp. 33$51,2001$.

[3] P. Ioannou and M. Stefanovic, "Evaluation of the ACC vehicles in mixed traffic:Lane change effects and sensitivity analysis," IEEE Trans. Intel. Transport. Syst.,vol. 6, no. 1, pp. 79-90, Jan. 2005.

[4] J. Zhang and P. Ioannou, "Longitudinal Control of Heavy Trucks:Environmental and Fuel Economy Considerations" IEEE Intel Trans Syst Conf.,Oct. 2004.

[5] M. Persson, F. Botling, E. Hesslow, and R. Johansson, "Stop and go controllerfor adaptive cruise control," in Proc. IEEE Int. Conf. Control Appl., 1999, pp. 1692-1697.

[6] S. Moon, I. Moon, and K.Yi, "Design, tuning, and evaluation of a full-rangeadaptive cruisecontrol system with collision avoidance." Control EngineeringPractice Vol 17 pp 442-455. 2009.

[7] S. Li, K. Li, R. Rajamani, and J. Wang, "Model predictive multi-objective vehicular adaptive cruise control" IEEE Trans. Control Syst. Technol., vol. 19, no. 3, may 2011.

[8] Dileep K, Sreepriya S, and Sreedeep Krishnan, "Vehicle dynamics and drive control for adaptive cruise vehicles" International Research Journal of Engineering and Technology (IRJET), Volume: 04 Issue: 12 | Dec-2017

[9] Li-hua LUO, Hong LIU, Ping LI, Hui WANG, "Model predictive control for adaptive cruise control with multi-objectives: comfort, fuel-economy, safety and car-following" Luo et al. / J Zhejiang Univ-Sci A (Appl Phys \& Eng) 2010 11(3):191-201

[10] R. Rajamani, Vehicle Dynamics and Control. New York: Springer Verlag, 2005.

[11] V.L. Bageshwar, W.L. Garrard, R. Rajamani. "Model Predictive Control of Transitional Maneuvers for Adaptive Cruise Control Vehicles", IEEE Transactions onVehicular Technology, 2004

[12] Shakouri P, Ordys A, Askari MR." Adaptive cruise control with stop\&go function using the state-dependent nonlinear model predictive control approach." doi: 10.1016/j.isatra.2012.05.001. Epub 2012 Jun 15

[13] Jiang, Y., Deng, W., He, R., Yang, S. et al., "Hierarchical Framework for Adaptive Cruise Control with Model Predictive Control Method," SAE Technical Paper 2017-01-1963, 2017.

[14] Liuping Wang, "Discrete Time Model Predictive Control Design using Laguerre Functions" Proceedings of the American Control Conference Arlington,VA June 25-27, 2001 